\title{
Plane Graphs with Parity Constraints
}

\author{
Oswin Aichholzer . Thomas Hackl . \\ Michael Hoffmann · Alexander Pilz · Günter Rote . \\ Bettina Speckmann · Birgit Vogtenhuber
}

the date of receipt and acceptance should be inserted later

\begin{abstract}
Let $S$ be a set of $n$ points in general position in the plane. Together with $S$ we are given a set of parity constraints, that is, every point of $S$ is labeled either even or odd. A graph $G$ on $S$ satisfies the parity constraint of a point $p \in S$ if the parity of the degree of $p$ in $G$ matches its label. In this paper, we study how well various classes of planar graphs can satisfy arbitrary parity constraints. Specifically, we show that we can always find a plane tree, a two-connected outerplanar graph, or a pointed pseudo-triangulation which satisfy all but at most three parity constraints. For triangulations we can satisfy about $2 / 3$ of the parity constraints and we show that in the worst case there is a linear number of constraints which cannot be fulfilled. In addition, we prove that for a given simple polygon $H$ with polygonal holes on $S$, it is NP-complete to decide whether there exists a triangulation of $H$ that satisfies all parity constraints.
\end{abstract}

Keywords triangulation $\cdot$ vertex degree parity $\cdot$ pseudo-triangulation $\cdot$ geometric graph

This research was initiated during the Fifth European Pseudo-Triangulation Research Week in Ratsch an der Weinstraße, Austria, 2008. Research of O. Aichholzer, T. Hackl, A. Pilz, and B. Vogtenhuber supported by the FWF [Austrian Fonds zur Förderung der Wissenschaftlichen Forschung] under grant S9205-N12, NFN Industrial Geometry. Research by B. Speckmann supported by the Netherlands Organisation for Scientific Research (NWO) under project no. 639.022.707.

O. Aichholzer · T. Hackl · A. Pilz · B. Vogtenhuber

Institute for Software Technology, Graz University of Technology, Austria.

E-mail: [oaich|thackl|apilz|bvogt] @ist.tugraz.at

M. Hoffmann

Institute for Theoretical Computer Science, ETH Zürich, Switzerland.

E-mail: hoffmann@inf.ethz.ch

G. Rote

Institut für Informatik, Freie Universität Berlin, Germany.

E-mail: rote@inf.fu-berlin.de

B. Speckmann

Dep. of Mathematics and Computer Science, TU Eindhoven, The Netherlands.

E-mail: speckman@win.tue.nl 


\section{Introduction}

Computing a simple graph that meets a given degree sequence is a classical problem in graph theory and theoretical computer science, dating back to the work of Erdôs and Gallai [8]. A degree sequence is a vector $d=\left(d_{1}, \ldots, d_{n}\right)$ of $n$ positive numbers. It is realizable iff there exists a simple graph whose nodes have precisely this sequence of degrees. Erdős and Gallai gave necessary and sufficient conditions for a degree sequence to be realizable, and several algorithms have been developed that generate a corresponding abstract graph.

An extension of this problem prescribes not only a degree sequence $d$, but also gives a set $S \subset \mathbb{R}^{2}$ of $n$ points in general position, where $p_{i} \in S$ is assigned degree $d_{i}$. It is well known that a degree sequence $d$ is realizable as a tree if and only if $\sum_{i=1}^{n} d_{i}=2 n-2$. Tamura and Tamura [19] extended this result to plane (straight line) spanning trees, giving an $O\left(n^{2} \log n\right)$ time embedding algorithm, which in turn was improved by Bose et al. [6] to optimal $O(n \log n)$ time.

In this paper we study a relaxation of this problem, where we replace exact degrees with degree parity: odd or even. Although parity constrains are significantly weaker than actual degree constrains, they still characterize certain (classes of) graphs. For example, Eulerian graphs are exactly those connected graphs where all vertices have even degree, and a classical theorem of Whitney states that a maximal planar graph is 3-colorable iff all vertices have even degree. A given graph might satisfy only a subset of the parity constraints. So we study how well various classes of planar graphs can satisfy arbitrary parity constraints. A preliminary version of this work has been presented at the Algorithms and Data Structures Symposium (WADS) in Banff, in August 2009 [1].

Definitions and notation. Let $S \subset \mathbb{R}^{2}$ be a set of $n$ points in general position. We denote the convex hull of $S$ by $\mathrm{CH}(S)$. The points of $S$ have parity constraints, that is, every point of $S$ is labeled either even or odd; for ease of explanation we refer to even and odd points. We denote by $n_{e}$ and $n_{o}$ the number of even and odd points in $S$, respectively. Throughout the paper an even point is depicted by $\boldsymbol{\Theta}$, an odd point by $\boldsymbol{\Phi}$, and a point that can be either by $\oplus$. A graph $G$ on $S$ makes a point $p \in S$ happy, if the parity of $\operatorname{deg}_{G}(p)$ matches its label. If $p$ is not happy, then it is unhappy. Throughout the paper a happy point is depicted by $\mathbf{O}$, an unhappy point by $\boldsymbol{O}$, and a point that can be either by $\boldsymbol{O}$.

Results. Clearly, not every set of parity constraints can be fulfilled. For example, in any graph the number of odd-degree vertices is even. Hence, the number of unhappy vertices has the same parity as $n_{o}$. For the class of plane trees, the aforementioned results on degree sequences immediately imply:

Theorem 1 On every point set $S \subset \mathbb{R}^{2}$ with parity constraints, there exists a plane spanning tree that makes $(i)$ all but two points happy if $n_{o}=0,\left(\right.$ ii) all but one point happy if $n_{o}$ is odd, and (iii) all points happy if $n_{o} \geq 2$ is even.

We show that we can always find a two-connected outerplanar graph (which is a Hamiltonian cycle with additional edges in the interior, Theorem 2) and a pointed pseudo-triangulation (Theorem 3), which satisfy all but at most three parity constraints. In Section 4 we consider triangulations. In Section 4.1 we show that if we are given a simple polygon $H$ with polygonal holes on $S$, it is NP-complete to decide whether there exists a triangulation of $H$ that satisfies all parity constraints. We show that there exist point sets and parity assignments such that the number of unhappy vertices grows linearly in $n$ for every triangulation on $S$. On the other hand, we can guarantee to satisfy about $2 / 3$ of the parity constraints 
(Theorem 7). This can be shown using results obtained from exhaustive computations on small point sets, and alternatively by a proof based on simple inductive constructions, that, however, sometimes involves elaborate case distinctions.

Related work. Many different types of degree restrictions for geometric graphs have been studied. For example, for a given set $S \subset \mathbb{R}^{2}$ of $n$ points, are there planar graphs on $S$ for which the maximum vertex degree is bounded? There clearly is a path, and hence a spanning tree, of maximum degree at most two. Furthermore, there is always a pointed pseudotriangulation of maximum degree five [13], although there are point sets where every triangulation must have a vertex of degree $n-1$. Another related question is the following: we are given a set $S \subset \mathbb{R}^{2}$ of $n$ points, together with a planar graph $G$ on $n$ vertices. Is there a plane straight-line embedding of $G$ on $S$ ? Outerplanar graphs are the largest class of planar graphs for which this is always possible, in particular, Bose [5] showed how to compute such an embedding in $O\left(n \log ^{2} n\right)$ time. Alvarez [4] considers the addition of extra (Steiner) points to make a triangulation of a planar point set 3-colorable (i.e., all inner vertices have even degree). For sets with $k$ interior points he proves that $\lfloor(k+2) / 3\rfloor$ Steiner points suffice. Fernández Delago et al. [9] issue triangulations of convex point sets with all vertices of even degree. They give the number of such triangulations and show that the graph of even triangulations obtained by exchanging the edges inside a hexagon is connected. They further prove the NP-completeness of the problem of extending a geometric graph to a 3-colorable triangulation by adding edges.

One motivation for our work on parity restrictions stems from a bi-colored variation of a problem stated by Erdôs and Szekeres in 1935: Is there a number $f^{\mathrm{ES}}(k)$ such that any set $S \subset \mathbb{R}^{2}$ of at least $f^{\mathrm{ES}}(k)$ bi-colored points in general position has a monochromatic subset of $k$ points that form an empty convex $k$-gon (that is, a $k$-gon that does not contain any points of $S$ in its interior)? It has been shown recently [2] that every bi-colored point set of at least 5044 points contains an empty (not necessarily convex) monochromatic quadrilateral. The proof uses, among others, a result that for any point set there exists a triangulation where at least half of the points have odd parity. Any increase in the guaranteed share of odd parity points translates into a lower minimum number of points required in the above statement. More specifically, from our Proposition 2 one can conclude that the above result holds for any set of at least 2080 points.

\section{Outerplanar Graphs}

After trees as minimally connected graphs, a natural next step is to consider two-connected graphs. In particular, outerplanar graphs generalize trees both in terms of connectivity and with respect to treewidth. In this section we consider two-connected outerplanar graphs, which are the same as outerplanar graphs with a unique Hamiltonian cycle [7], in other words, simple polygons augmented with a set of pairwise non-crossing diagonals.

The following simple construction (see (Fig. 1) makes all but at most three points happy. Pick an arbitrary point $p$. Set $p_{1}=p$ and denote by $p_{2}, \ldots, p_{n}$ the sequence of points from $S$, as encountered by a counterclockwise radial sweep around $p$, starting from some suitable direction (if $p$ is on $\mathrm{CH}(S)$ towards its counterclockwise neighbor on $\mathrm{CH}(S)$ ). The outerplanar graph $G$ consists of the closed polygonal chain $P=\left(p_{1}, \ldots, p_{n}\right)$ plus an edge $p p_{j}$ for every odd point $p_{j} \in\left\{p_{3}, \ldots, p_{n-1}\right\}$. All points are happy, with the possible exception of $p, p_{2}$, and $p_{n}$. Fig. 1 shows an example of a point set $S$ with parity constraints and an outerplanar graph on $S$ such that all but two points are happy. 

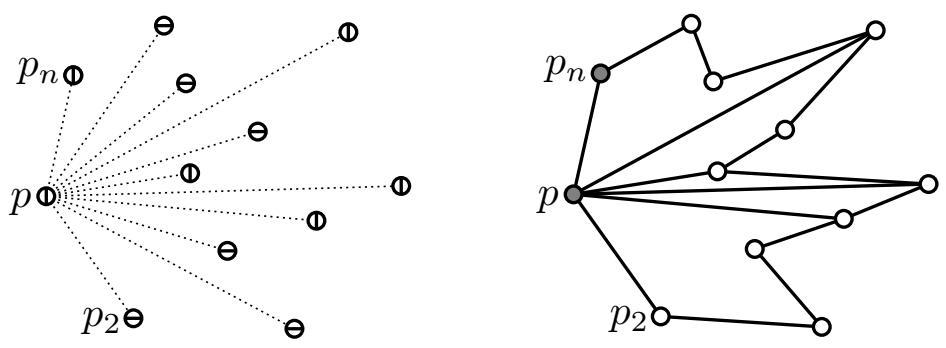

Fig. 1 Constructing a two-connected outerplanar graph with at most three unhappy vertices.

Theorem 2 For every set $S \subset \mathbb{R}^{2}$ of $n$ points with parity constraints, there exists an outerplanar graph on $S$ that makes all but at most three points happy.

\section{Pointed Pseudo-Triangulations}

Pseudo-triangulations are related to triangulations and use pseudo-triangles in addition to triangles. A pseudo-triangle is a simple polygon with exactly three interior angles smaller than $\pi$. A pseudo-triangulation is called pointed if every vertex $p$ has one incident region whose angle at $p$ is greater than $\pi$. In the following we describe a recursive construction for a pointed pseudo-triangulation $\mathscr{P}$ on $S$ that makes all but at most three points of $S$ happy.

At any time in our construction we have only one recursive subproblem to consider. This subproblem consists of a point set $S^{*}$ whose convex hull edges have already been added to $\mathscr{P}$. The current set $\mathscr{P}$ is a pointed set of edges that subdivides the exterior of $\mathrm{CH}\left(S^{*}\right)$ into pseudo-triangles such that all points outside $\mathrm{CH}\left(S^{*}\right)$ are happy. $\mathscr{P}$ contains no edges inside $\mathrm{CH}\left(S^{*}\right)$. We say that $\mathrm{S}^{*}$ is hopeful if at least one point on $\mathrm{CH}\left(S^{*}\right)$ is made happy by the current version of $\mathscr{P}$. Otherwise, we say that $S^{*}$ is unhappy.

We initialize our construction by setting $S^{*}=S$ and adding $\mathrm{CH}(S)$ to $\mathscr{P}$. Now we distinguish four cases.

(1) $S^{*}$ is hopeful. Let $v$ be a point on $\mathrm{CH}\left(S^{*}\right)$ that is currently happy, let $p$ and $q$ be its neighbors, and let $S^{\prime}$ be the (possibly empty) set of points from $S$ that lie in the interior of the triangle $\triangle_{q v p}$. Then $\mathrm{CH}\left(S^{\prime} \cup\{p, q\}\right)$ without the edge
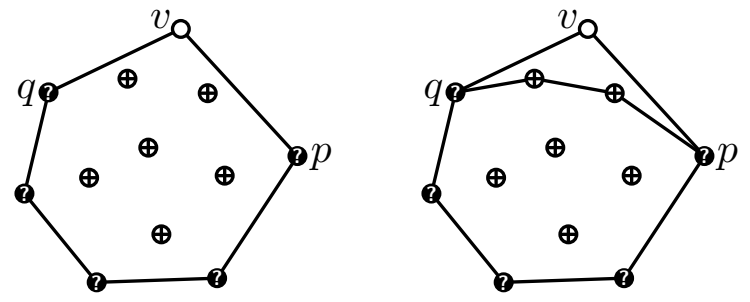
$p q$ defines a convex chain $C$ from $p$ to $q$, in a way that $C$ and $v$ together form a pseudotriangle. (If $S^{\prime}=\emptyset$, then $C=p q$.) Remove $v$ from consideration by adding $C$ to $\mathscr{P}$. If $\left|S^{*}\right| \geq 5$, recurse on $S^{*} \backslash\{v\}$. Otherwise, there are at most three unhappy points in the remaining triangle.

(2) $S^{*}$ is unhappy and has no interior points. Choose one point $p$ on $\mathrm{CH}\left(S^{*}\right)$ and triangulate $\mathrm{CH}\left(S^{*}\right)$ by adding edges from $p$. There are at most three unhappy points, namely $p$ and its two neighbors.
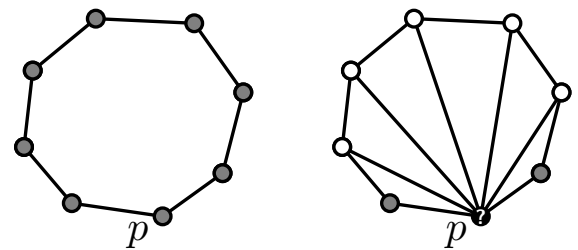
(3) $S^{*}$ is unhappy and has exactly one interior point, $p_{i}$. Pick an arbitrary point $p$ on $\mathrm{CH}\left(S^{*}\right)$ and draw a line through $p$ and $p_{i}$. This line intersects exactly one edge $e$ of $\mathrm{CH}\left(S^{*}\right)$, and $e, p$, and $p_{i}$ together define a pseudotriangle $\nabla$. Add $\nabla$ to $\mathscr{P}$, which splits $\mathrm{CH}\left(S^{*}\right)$
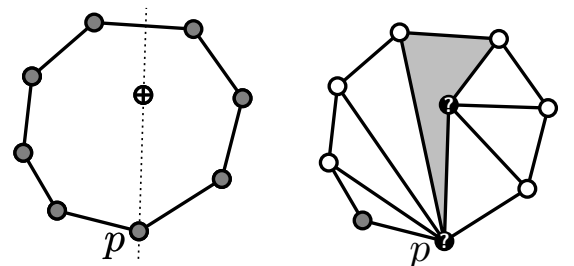
into two sub-polygons. Triangulate the sub-polygon which contains $p_{i}$ by adding edges from $p_{i}$ to all other vertices, except to its neighbors. Similarly, triangulate the other subpolygon by adding edges from $p$. There are at most three unhappy points: $p, p_{i}$, and a neighbor of $p$.

(4) $S^{*}$ is unhappy and has more than one interior point. Let $S_{i}$ be the set of interior points. First add the edges of $\mathrm{CH}\left(S_{i}\right)$ to $\mathscr{P}$. Then connect each point on $\mathrm{CH}\left(S^{*}\right)$ tangentially to $\mathrm{CH}\left(S_{i}\right)$ in clockwise direction, thereby creating a "lens shutter" pattern. Each point on
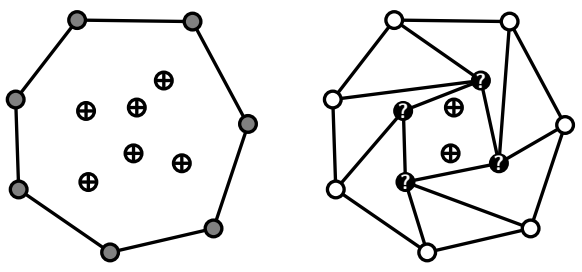
$\mathrm{CH}\left(S^{*}\right)$ is now happy. If $\left|S_{i}\right|>3$, then recurse on $S_{i}$. Otherwise, there are at most three unhappy points.

Theorem 3 For every point set $S \subset \mathbb{R}^{2}$ with parity constraints, there exists a pointed pseudo-triangulation on $S$ that makes all but at most three points of S happy.

\section{Triangulations}

The final and maybe most interesting class of planar graphs which we consider are triangulations. If the point set $S$ lies in convex position, then all pseudo-triangulations of $S$ are in fact triangulations. Thus, Theorem 3 also holds for triangulations of convex point sets. Moreover, we may select any three points $p, q, r$ that are consecutive along $\mathrm{CH}(S)$, which we do not remove when the set is hopeful. When no points can be removed, we complete the triangulation by adding edges to $q$. This immediately gives the following result.

Corollary 1 For every point set $S \subset \mathbb{R}^{2}$ in convex position with parity constraints, and any three points $p, q, r$ that are consecutive along $\mathrm{CH}(S)$, there exists a triangulation on $S$ that makes all points of $S$ happy, with the possible exception of $p, q$, and $r$.

In contrast to such a triangulation of a convex domain, it is easy to construct arbitrary large examples of simple polygons that do not even have one happy vertex. In the following, we consider complexity aspects of triangulating polygons with parity constraints. After that, we give bounds on the number of happy vertices in triangulations of point sets.

\subsection{Triangulations of Polygons}

It is a well-known and easy fact that there always exists a proper vertex 3-coloring of any triangulation of a simple polygon [17, p. 15]. There also is an interesting connection between proper 3-colorings and the parity of the vertices.

Theorem $4([\mathbf{1 0 , 1 5 ] )}$ Given a triangulation $T(P)$ of a simple polygon $P$ let $u, v$, and $w$ be any three consecutive vertices of $P$. Then, in a proper vertex 3-coloring of $T(P)$, the vertices $u$ and $w$ have the same color if and only if $v$ is odd. 
This follows from the fact that in the sequence of vertices that are neighbors to $v$ in the triangulation their colors must alternate. Fleischner [10] actually proves this for the more general case that allows inner vertices of even degree. Kooshesh and Moret [15] describe a trivial algorithm for coloring a triangulated polygon in linear time that immediately follows from the above theorem. Indeed, Theorem 4 gives a simple test for checking whether a simple polygon can be happily triangulated: Start with two arbitrary colors for two adjacent vertices, and propagate the 3-coloring along the boundary, using Theorem 4. A happy triangulation exists if and only if this results in a proper 3-coloring of the vertices.

Optimal triangulations of arbitrary simple polygons can be computed in $O\left(n^{3}\right)$ time by adapting the well-known dynamic-programming approach of $[11,14]$ (devised for the minimum-weight triangulation problem), where each triangle that can be incident to a chosen base edge defines two subproblems. As by combining two subproblems the parity of their common vertex might change, optimal partial solutions are stored for all four different parity patterns at the base edge of a subproblem.

In contrast, the situation gets more involved if we consider polygons with holes.

Theorem 5 It is NP-complete to decide, for a given polygon $H$ with holes and with parity constraints, whether there exists a triangulation of $H$ such that all vertices of $H$ are happy.

Proof Following Jansen [12], we use a restricted version of the NP-complete planar 3-SAT problem [16], in which each clause contains at most three literals and each variable occurs in at most three clauses.
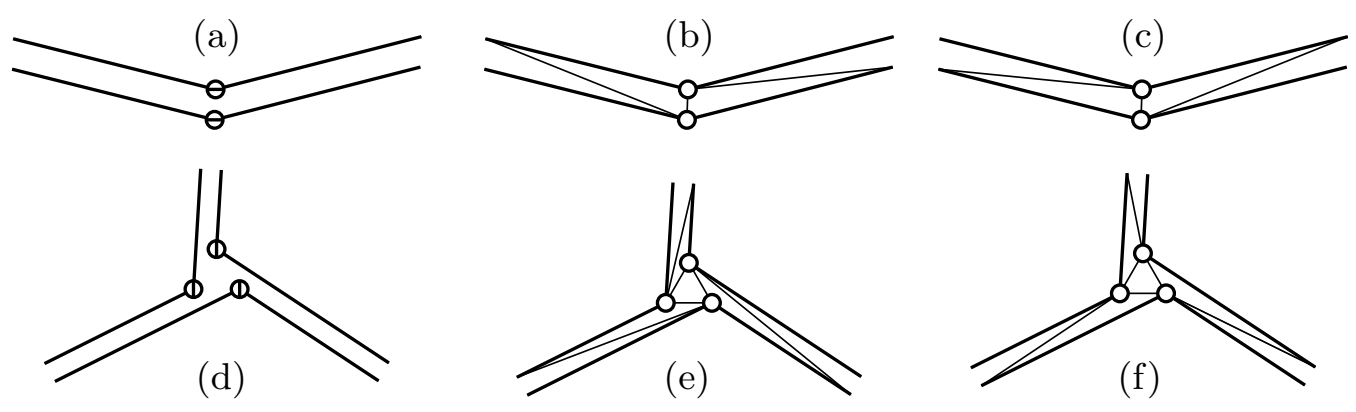

Fig. 2 A wire (a) that transfers tRUe (b), and FAlSE (c), and a variable (d) in TRUE (e) and FAlSE (f) state. The short edges are part of in every triangulation.

The edges of the planar formula are represented by wires (Fig. 2(a)-(c)), narrow corridors which can be triangulated in two possible ways, and thereby transmit information between their ends. Negation can easily be achieved by swapping the labels of a single vertex pair in a wire from both even to both odd. The construction of a variable (Fig. 2(d)-(f)) ensures that all wires emanating from it carry the same state, that is, their diagonals are oriented in the same direction.

To check clauses we use an OR-gate (Fig. 4) with two inputs and one output wire. The OR-gate is a convex 9-gon $v_{1} \ldots v_{9}$ with three attached wires, and a don't-care loop (Fig. 3(a)) attached to the two top-most vertices $v_{8}, v_{9}$. This loop has two possible triangulations and gives more freedom for the two vertices to which it is attached: by switching between the two triangulations of the loop the parity of both vertices is changed. All edges of the 9-gon are either on the boundary of the input polygon or they are unavoidable: no other potential edge crosses them, and thus they must belong to every triangulation. This can be achieved by making them short enough. Starting at the leftmost vertex $v_{1}$ (see Fig. 4(a)), the 


\begin{tabular}{|r||c|c|c|c|c|c|c|c|c|c|}
\hline index & 1 & 2 & 3 & 4 & 5 & 6 & 7 & 8 & 9 & $10 \simeq 1$ \\
\hline \hline$\lambda_{f}$ & $o$ & $e$ & $e$ & $e$ & $o$ & $e$ & $e$ & $o$ & $o$ & $o$ \\
\hline color & 1 & 2 & 3 & 1 & 2 & 1 & 3 & 2 & 3 & $2 \neq 1$ \\
\hline \hline$\lambda_{f}^{\prime}$ & $o$ & $e$ & $e$ & $e$ & $o$ & $e$ & $e$ & $e$ & $e$ & $o$ \\
\hline color & 1 & 2 & 3 & 1 & 2 & 1 & 3 & 2 & 1 & $3 \neq 1$ \\
\hline
\end{tabular}

Table 1 Invalid colorings induced by the vertex constraints show the nonexistence of a triangulation of the OR-gate with both inputs FALSE and the output TRUE.

constraint sequence of the vertices in counterclockwise order is $\lambda=\langle$ oeoeeoeoe $\rangle$, where $e$ stands for even and $o$ for odd.

Fig. 4 shows triangulations of the OR-gate for the four possible input configurations of an OR-gate, where the output is FALSE iff both inputs are false. There may also be triangulations of an OR-gate such that the output can be FALSE even if one input is TRUE. The important part is that (i) when at least one input is TRUE, there is a triangulation with output TRUE, see Fig. 4(b-d), and (ii) if both inputs are FALSE, the output must also be FALSE.

Suppose the inputs are both FALSE and the output is TRUE. Remove the edges outside of the 9-gon and adjust the labeling of the the 9-gon accordingly. We get $\lambda_{f}=\langle$ oeeeoeeoo $\rangle$, and for a different direction of the don't-care loop $\lambda_{f}^{\prime}=\langle$ oeeeoeeee $\rangle$. If we apply the test of Theorem 4 and try to 3 -color the vertices, as shown in Table 1, we get a conflict, and hence there is no triangulation with the given parities.

Clauses with two literals can directly be realized by such gates, three literals require to cascade two OR-gates (Fig. 3(b)). In both cases, we fix the output to TRUE by simply removing the output wire and swapping the parity of the 6-th vertex $v_{6}$.

It is straightforward to combine the constructed elements to a polygon $H$ with holes representing a given planar 3SAT formula.

\subsection{Triangulations of Point Sets}

In this section we present lower and upper bounds on the number of happy vertices for general point sets. For example, for point sets of small cardinality we can investigate the number of happy vertices with the help of the order type data base [3]. For any set of 11 points with parity constraints we can always find a triangulation which makes at least 7 vertices happy, cf. Table 2 in Section 4.2.2.

\subsubsection{A Lower Bound on Unhappy Vertices}

The figure below shows a double circle for 10 points with parity constraints, such that at most 5 points can be made happy. This is in fact the only point configuration for $n=10$ (out

(a)

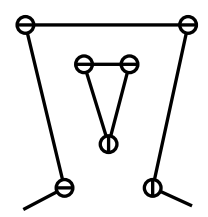

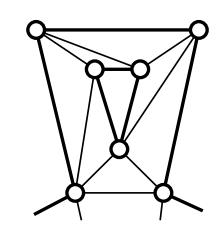

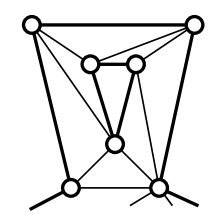

(b)

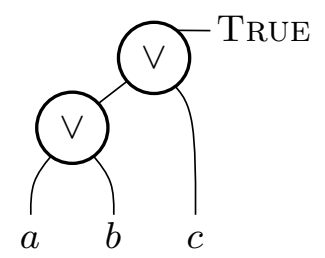

Fig. 3 A don't-care loop (a). Checking a clause $a \vee b \vee c$ by joining two OR-gates (b). 


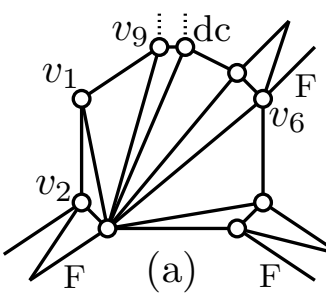

(a)

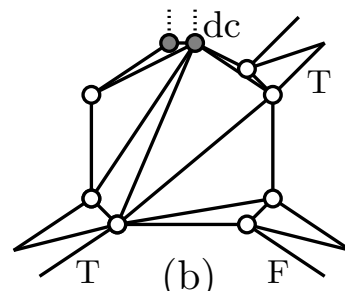

(b)

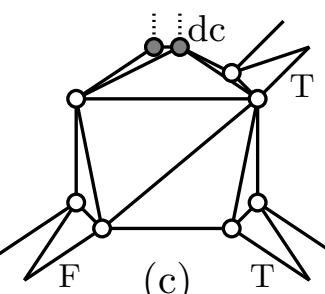

(c)

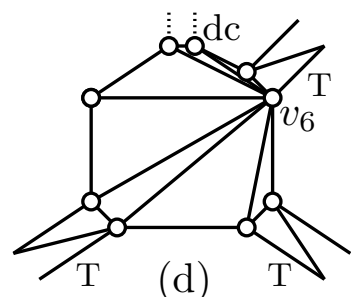

(d)

Fig. 4 An Or-gate with inputs False, False (a), True, False (b), False, True (c), and True, True (d). The two inputs are at the bottom and the output is at the upper right side. A don't-care loop "dc" is attached to the two top-most vertices.

of 14309547 [3]) with this property. A double circle of even size $n=2 h$ is a point set with $h$ extreme vertices in which each of the remaining $h$ interior points is placed sufficiently close to a different edge of the convex hull. For each interior point, the edges to the two adjacent hull vertices are unavoidable; they are part of every triangulation.

These unavoidable edges form a polygon Therefore, triangulating the interior of the double circle is equivalent to triangulating a simple polygon. This allows examining the double circle using the already mentioned dynamic-programming approach without explicitly generating geometric representations.
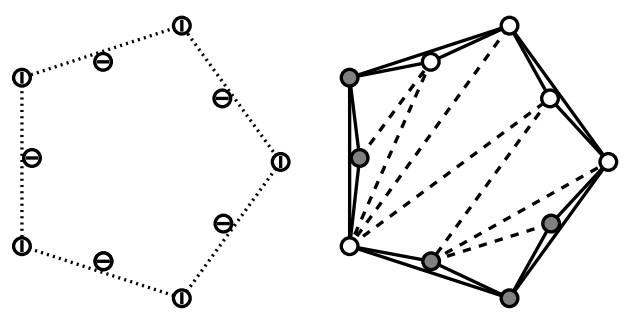

Based on the double circle we constructed large examples with a repeating parity pattern $\sigma=\left\langle\left(e e(o e)^{3} e e(o e)^{7} e e(o e)^{5}\right)^{3}\right\rangle$ of length 108, starting at an extreme vertex and proceeding counterclockwise. We will show that for these configurations any triangulation has at least $n / 108+2$ unhappy vertices. Our proof uses computer aid. An extensive discussion of the proof and its underlying parity pattern can be found in the master's thesis of one of the authors [18].

The proof is inspired by the dynamic-programming approach of combining two subpolygons which are separated by a triangle and for which the minimum number of unhappy vertices has already been determined. The proof works by induction over the size of the subproblem. Consider a double circle of size $n=|\sigma| \cdot s$, labeled with $s$ repetitions of $\sigma$. We call a sequence of points labeled by such a repetition a $\sigma$-instance. Add the unavoidable edges and remove the convex hull edges. Let the resulting polygon be called a double circle polygon.

Consider a diagonal $d$ from the $i$-th vertex in a $\sigma$-instance to the $j$-th vertex in the $k$ th following $\sigma$-instance in the counterclockwise direction, see Fig. 5. (For $k=0$, the two vertices are taken from the same $\sigma$-instance. These diagonals will form the fixed-size subproblems.) We denote by $f_{i j}(k)$ the minimum possible mumber of unhappy vertices in a triangulation of the polygon formed by $d$ and the vertices between the endpoints of $d$. For small values of $k$, these numbers can be explicitly calculated with a dynamic-programming recursion. We make a claim of the following form:

$$
\begin{array}{ll}
f_{i j}(0)=\kappa_{i j}, & \text { for } 1 \leq i<j \leq|\sigma|, \\
f_{i j}(k) \geq c_{i j}+k, & \text { for } k \geq 1,1 \leq i, j \leq|\sigma|,
\end{array}
$$

for constants $\kappa_{i j}$ and $c_{i j}$.

Our goal is to prove (2) by induction on the number of vertices between the endpoints of $d$. In the triangulations over which we optimize for the subproblem $f_{i j}(k)$, for $k \geq 1$, consider 
the triangle with base edge $d$. It can partition the subproblem $f_{i j}(k)$ in three ways, see Fig. 5: its apex $v_{m}$ is either in the starting $\sigma$-instance or in the ending $\sigma$-instance (together with one of the endpoints of $d$ ), or in some intermediate $\sigma$-instance. We must take the minimum of these cases. When disregarding for a moment the parity of $d$ 's end vertices, we get:

$$
\begin{aligned}
& f_{i j}^{1}(k)=\min _{i<m_{1} \leq|\sigma|}\left[f_{i m_{1}}(0)+f_{m_{1} j}(k)\right]=\min _{i<m_{1} \leq|\sigma|}\left[\kappa_{i m_{1}}+f_{m_{1} j}(k)\right], \\
& f_{i j}^{2}(k)=\min _{1 \leq m_{2}<j}\left[f_{i m_{2}}(k)+f_{m_{2 j}}(0)\right]=\min _{1 \leq m_{2}<j}\left[f_{i m_{2}}(k)+\kappa_{m_{2} j}\right], \\
& f_{i j}^{3}(k)=\min _{1 \leq m_{3} \leq|\sigma|, 0<l<k}\left[f_{i m_{3}}(l)+f_{m_{3} j}(k-l)\right] \\
& f_{i j}(k)=\min \left\{f_{i j}^{1}(k), f_{i j}^{2}(k), f_{i j}^{3}(k)\right\} .
\end{aligned}
$$
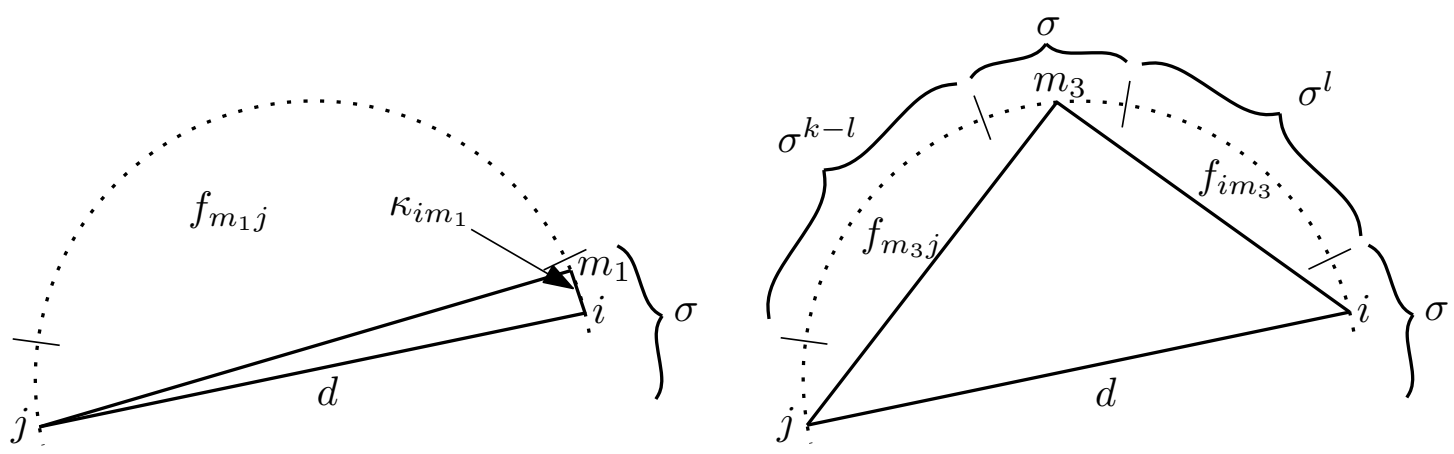

Fig. 5 The different types of subproblems formed by triangles with their base at $d$.

The simplified hypothesis (2) which we want to prove by induction over the size of the subproblem is that $f_{i j}(k) \geq k+c_{i j}$ for some constant $c_{i j}$. The induction hypothesis (1-2) gives

$$
\begin{aligned}
& f_{i j}^{1}(k) \geq \min _{i<m_{1} \leq|\sigma|}\left[\kappa_{i m_{1}}+k+c_{m_{1} j}\right], \\
& f_{i j}^{2}(k) \geq \min _{1 \leq m_{2}<j}\left[k+c_{i m_{2}}+\kappa_{m_{2} j}\right], \\
& f_{i j}^{3}(k) \geq \min _{1 \leq m_{3} \leq|\sigma|, 0 \leq l<k}\left[l+c_{i m_{3}}+k-l+c_{m_{3} j}\right] .
\end{aligned}
$$

To prove $f_{i j}(k) \geq k+c_{i j}$ for $k \geq 1$ it therefore suffices to show that

$$
\begin{array}{rlrl}
\kappa_{i m_{1}}+k+c_{m_{1} j} & \geq k+c_{i j} & & \forall m_{1}, i<m_{1} \leq|\sigma| \\
k+c_{i m_{2}}+\kappa_{m_{2} j} \geq k+c_{i j} & & \forall m_{2}, 1 \leq m_{2}<j \\
l+c_{i m_{3}}+k-l+c_{m_{3} j} \geq k+c_{i j} & & \forall m_{3}, 1 \leq m_{3} \leq|\sigma|, \forall k, l .
\end{array}
$$

These inequalities obviously allow us to disregard the variables $l$ and $k$. We only need to compare the constants.

Let us now take the parity of end vertices of the diagonal into account. Let $f_{i j}^{h h}(k)$ define the least number of unhappy vertices in the subproblem with $k+1 \sigma$-instances and with both end vertices happy, and let $f_{i j}^{u h}(k), f_{i j}^{h u}(k)$ and $f_{i j}^{u u}(k)$ be defined analogously with the first, the second and both end vertices unhappy, respectively. Similarly, we extend the notion for fixed-size subproblem minima to $\kappa_{i j}^{h h}, \kappa_{i j}^{u h}, \kappa_{i j}^{h u}$ and $\kappa_{i j}^{u u}$. By convention, we do not include the number of unhappy end vertices in $f_{i j}^{p q}(k)$ and $\kappa_{i j}^{p q}$. Further note that some of the fixed-size subproblems may not exist. Inequalities containing them do not impose a 
valid subproblem and therefore need not be checked. When combining two subproblems, they have a common vertex at the apex $v_{m}$. If it is happy in one subproblem and unhappy in the other, the combined degree is odd. Hence, we increment the number of unhappy vertices if $v_{m}$ is labeled even (recall that $v_{m}$ has not been counted before). Otherwise, if $v_{m}$ has the same state of happiness in both subproblems, the combined degree is even. Therefore, we increment the number of unhappy vertices if $v_{m}$ is labeled odd. Further, the addition of $d$ changes the parity of its end vertices. For, e.g., $f_{i j}^{h h}$ we therefore have to consider the combinations of subproblems that have unhappy vertices at $i$ and $j$. Let $L(m)=1$ if the $m$-th label in $\sigma$ is odd and $L(m)=0$ otherwise. We now have to prove for, e.g., $f_{i j}^{h h}$

$$
\left.\begin{array}{rl}
\kappa_{i m_{1}}^{u u}+c_{m_{1} j}^{u u}+L\left(m_{1}\right) & \geq c_{i j}^{h h} \\
\kappa_{i m_{1}}^{u u}+c_{m_{1} j}^{h u}+1-L\left(m_{1}\right) & \geq c_{i j}^{h h} \\
\kappa_{i m_{1}}^{u h}+c_{m_{1} j}^{u u}+1-L\left(m_{1}\right) & \geq c_{i j}^{h h} \\
\kappa_{i m_{1}}^{u h}+c_{m_{1} j}^{h u}+L\left(m_{1}\right) & \geq c_{i j}^{h h}
\end{array}\right\} \forall m_{1}, i<m_{1} \leq|\sigma|
$$

The inequalities for $f_{i j}^{h u}, f_{i j}^{u h}$ and $f_{i j}^{u u}$ are analogous.

As mentioned above, a dynamic-programming recursion can explicitly calculate the $f_{i j}^{h h}(k), f_{i j}^{h u}(k), f_{i j}^{u h}(k)$, and $f_{i j}^{u u}(k)$ for small values of $k$. This gives us the values of $\kappa_{i j}^{p q}$ and it allows us to guess the values for the constants $c_{i j}^{p q}$, for all combinations of happiness labels $p, q$. Once these constants are found, we just have to check the inequalities (3-5), again using a computer program.

However, it turned out that this setup did not lead to a valid proof. We have to refine the inductive claim (1-2) by treating also the case $k=1$ as a "fixed-size" problem:

$$
\begin{aligned}
& f_{i j}(0)=\kappa_{i j}, \quad \text { for } 1 \leq i<j \leq|\sigma|, \\
& f_{i j}(1)=\kappa_{i,|\sigma|+j}, \quad \text { for } 1 \leq i, j \leq|\sigma| \text {, } \\
& f_{i j}(k) \geq c_{i j}+k, \quad \text { for } k \geq 2,1 \leq i, j \leq|\sigma|,
\end{aligned}
$$

The inequalities have to be modified accordingly. For example, we have to add assertions for the two following inequalities (again simplified, without taking into account the states $p, q$ of the boundary vertices).

$$
\begin{array}{ll}
\kappa_{i m}+c_{m-|\sigma|, j}-1 \geq c_{i j} & \forall m,|\sigma|<m \leq 2|\sigma| \\
c_{i m}-1+\kappa_{m,|\sigma|+j} \geq c_{i j} & \forall m, 1 \leq m \leq|\sigma| .
\end{array}
$$

In both inequalities we have to subtract 1 on the left side, because the non-fixed-size subproblem has now size $k-1$ and the fixed-size subproblem extends over two $\sigma$-instances. Taking $c_{i j}^{p q}:=f_{i j}^{p q}(2)-2$, all inequalities in this modified setting are now satisfied, establishing that our polygon with $n=s \cdot|\sigma|=108 s$ vertices makes at least $s+2$ vertices unhappy: 
Theorem 6 The maximum number of unhappy vertices in the best triangulations of all point sets of size $n$ with parity constraints is $\Theta(n)$.

Open Problem 1 in [2] asks for the maximum constant $c$ such that for any point set there always exists a triangulation where $c n-o(n)$ points have odd degree. While for the question as stated we still believe that $c=1$ is possible, the above construction shows (using the double circle) that for general parity constraints we have $c \leq \frac{107}{108}$.

The upper bound on $c$ can be improved to $\frac{98}{99}$ by removing the nine even extremal vertices of $\sigma$ and flipping the labels of the neighboring vertices. The triangulations of the resulting smaller polygon $P^{\prime}$ with $99 s$ vertices are in one-to-one correspondence with those triangulations of the original polygon $P$ in which the removed vertices form ears (degree-2 vertices) and are thus happy. Since the original polygon $P$ with $108 s$ vertices has no triangulation with more than $107 s$ happy vertices, it is clear that $P^{\prime}$ has no triangulation with more than $98 s$ happy vertices.

\subsubsection{A Lower Bound on Happy Vertices}

As already mentioned, using the order type data base [3] we have investigated point sets of small cardinality by computer. Table 2 shows the values $\max _{|S|=n} \max _{\lambda} \min _{T} u(T, \lambda)$, where $u(T, \lambda)$ is the number of unhappy vertices in a triangulation $T$ of a point set $S$ for parity constraints $\lambda$. For all-odd and all-even, respectively, the $\max _{\lambda}$-term is replaced by parity constraints such that all vertices have to be odd (even). Similarly for all-inner-odd and allinner-even all the inner vertices have to be odd (even), and for the extremal vertices we take the worst parity constraints.

\begin{tabular}{|l||c|c|c|c|c|c|c|c|c|}
\hline$n$ & 3 & 4 & 5 & 6 & 7 & 8 & 9 & 10 & 11 \\
\hline \hline worst parity constraints & 3 & 4 & 3 & 4 & 4 & 4 & 4 & 5 & 4 \\
\hline all odd & 3 & 2 & 3 & 2 & 3 & 2 & 3 & 2 & 3 \\
\hline all even & 0 & 4 & 2 & 4 & 2 & 4 & 4 & 4 & 4 \\
\hline all inner odd & 3 & 3 & 3 & 3 & 3 & 3 & 3 & 3 & 3 \\
\hline all inner even & 3 & 4 & 3 & 4 & 4 & 4 & 4 & 5 & 4 \\
\hline
\end{tabular}

Table 2 Maximum number of unhappy vertices in the best triangulation of a set of $n$ points with the described parity constraints, $n \leq 11$

It is noteworthy that the all-inner-even cases already give the worst bounds among all parity constraints. (In line with this observation, the bad labeling that we chose for the double-circle in the previous section had indeed all inner vertices even.) In contrast, the all-inner-odd case never causes more than 3 unhappy vertices.

The results of Table 2 allow a simple construction for a lower bound on the number of happy vertices.

Proposition 1 For every set $S \subset \mathbb{R}^{2}$ of $n$ points with parity constraints, there exists a triangulation on $S$ that makes at least $8\left\lfloor\frac{n}{12}\right\rfloor-1$ points happy.

Proof Given a point set $S$, select an extreme vertex $p$ and radially sort the remaining $n-1$ vertices around $p$. We call every twelfth vertex in this order a separating vertex. The lines through $p$ and every separating vertex around it split groups $G_{i}$ of eleven points (probably less in the last group). Construct the convex hull boundary for each of these groups. We show 

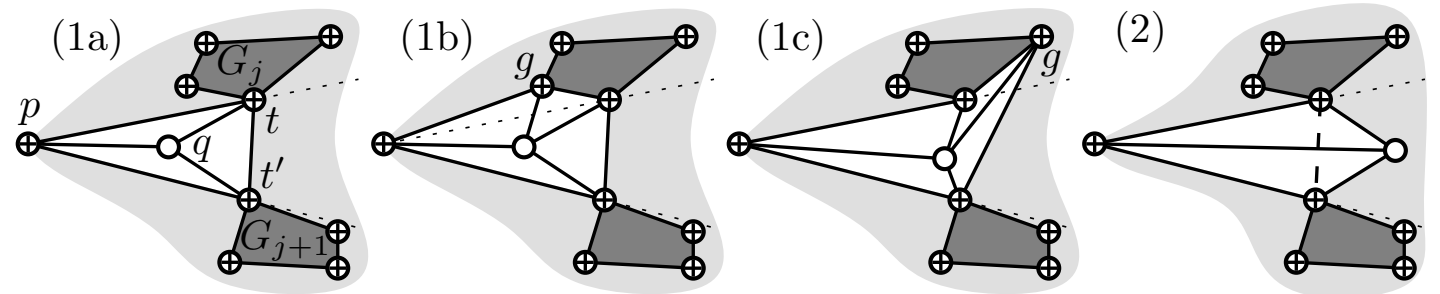

Fig. 6 Construction for the lower bound using the order type data base results. The gray regions depict the convex hulls of groups of eleven points. The three different cases to handle the separating vertices are shown.

that there always exists a triangulation of $D=\mathrm{CH}(S) \backslash \bigcup_{i} \mathrm{CH}\left(G_{i}\right)$ such that all separating vertices are happy. Consider a separating vertex $q$, and let its two neighboring groups be $G_{j}$ and $G_{j+1}$. Further, let $t$ and $t^{\prime}$ be the predecessor and successor of $q$ in the order around $p$, respectively, see Fig. 6.

(1) The separator $q$ is inside the triangle $p t^{\prime} t$. If $q$ is labeled odd, we draw edges between each of these four vertices, see Fig. 6(1a). If $q$ is labeled even, let $g$ be a neighbor of $t$ on $\mathrm{CH}\left(G_{j}\right)$ which is visible from $q$. Draw the quadrilateral $p t^{\prime} t g$ (or $p t^{\prime} g t$ ) and draw the edges from $q$ to all of them, see Fig. 6(1b-1c).

(2) The quadrilateral $p t^{\prime} q t$ is convex. Draw the quadrilateral and the edge $p q$. If, after triangulating the rest of $D, q$ is unhappy, exchange the edge $p q$ by the edge $t t^{\prime}$ to make $q$ happy.

According to Table 2 we can make all but 4 vertices happy in each group of 11 . Let $n \equiv k$ $(\bmod 12)$. We have $\frac{n-k}{12}$ full groups containing at least 7 happy vertices each, and $\frac{n-k}{12}-1$ happy separating vertices. The vertex $p$ and the $k$ remaining vertices after the last full group might be unhappy. ${ }^{1}$ Thus, we have at least $7 \frac{n-k}{12}+\frac{n-k}{12}-1=8 \frac{n-k}{12}-1=8\left\lfloor\frac{n}{12}\right\rfloor-1$ happy vertices.

Proposition 2 For any point set $S$ of size $n$ with all vertices labeled odd, there exists a triangulation making at least $10\left\lfloor\frac{n}{13}\right\rfloor-2$ vertices happy.

Proof The proof uses the same technique and notation as the one of Proposition 1. Instead of one vertex $q$ we now use two vertices $a$ and $b$ between groups of 11 points and show that we can always make $a$ and $b$ odd, see Fig. 7. We consider three different cases.

(1) If $a, b, p, t$, and $t^{\prime}$ are in convex position, after triangulating the exterior, $a$ and $b$ can be made happy due to Corollary 1.

(2) If both, $a$ and $b$, are inside of the triangle $p t^{\prime} t$, remove $b$ and make $a$ even as in the proof of Proposition 1, Case (2). Add $b$ again. It is now inside a triangle that is incident to $a$. Draw the edges between $b$ and all the vertices of the triangle. Both $a$ and $b$ are now odd.

(3) If w.l.o.g. $a, t, p$, and $t^{\prime}$ form a convex quadrilateral, we distinguish between two subcases.

(3.1) Suppose $b$ is inside of the triangle $a t t^{\prime}$. Remove $b$ and make $a$ even like in the proof of Proposition 1, Case (3). Then add $b$ again and draw the edges to the vertices of the triangle containing it. One of these vertices is $a$ that now becomes happy.

(3.2) Vertex $b$ is inside the triangle $p t^{\prime} t$. There exists a vertex $g$ next to $t$ on $\mathrm{CH}\left(G_{j}\right)$ that is visible to $b$. Form a (not necessarily convex) 5 -gon by adding $g$ to the quadrilateral in a radial order around $b$.

1 Depending on $k$ we could perform better for the vertices of the last group, but this would only give a marginal improvement of the additive factor, while making the bound dependent on $k$. 

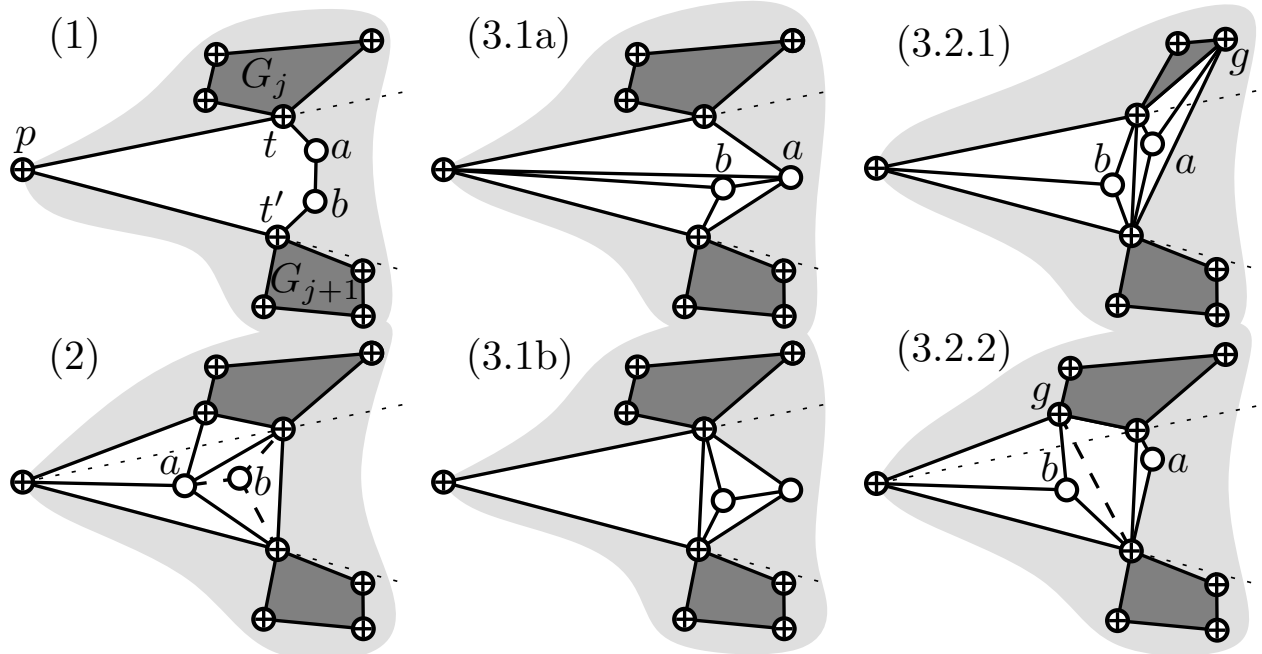

Fig. 7 Two vertices between two groups can be made odd. Examples for the different cases are shown, as well as the two possibilities for Case (3.1). The dashed stroke for Case (3.2.2) depicts the flipped edge.

(3.2.1) If $a$ is a reflex vertex, draw the edge $t^{\prime} g$ that is outside of the 5-gon. Draw the edges $a t$ and $t t^{\prime}$, as well as the edges from $b$ to $p, t$, and $t^{\prime}$.

(3.2.2) If $a$ is a convex vertex of the 5-gon, triangulate the exterior. If $a$ is unhappy, draw all edges from $b$ to the vertices of the 5-gon. If $a$ is happy, draw the edge between $t$ and $a$ 's neighbor (which is either $t$ or $g$ ). Add all edges from $b$ to the remaining vertices. Since $b$ is of degree four, one of the edges incident to it can be flipped (i.e., the edge is removed and the other diagonal of the resulting convex 4-gon is added). After the flip, $b$ has degree 3 and $a$ remains happy.

The bound calculated in Proposition 1 improves to $10\left\lfloor\frac{n}{13}\right\rfloor-2$ happy vertices for all-odd constraints, using the all-inner-odd result from Table 2.

Alternatively to the bound construction using the order type data base, we also give a stand-alone construction for the general case. The following simple observation will prove to be useful.

Observation 1 For every set $S \subset \mathbb{R}^{2}$ of four points in convex position with parity constraints and every $p \in S$ there exists a triangulation on $S$ that makes at least two of the points from $S \backslash\{p\}$ happy.

Theorem 7 For every set $S \subset \mathbb{R}^{2}$ of $n$ points with parity constraints, there exists a triangulation on $S$ that makes at least $\left\lfloor\frac{2 n}{3}\right\rfloor-6$ points of $S$ happy.

Proof Pick an arbitrary point $p$ on $\mathrm{CH}(S)$, set $p_{1}=p$, and denote by $p_{2}, \ldots, p_{n}$ the sequence of points from $S$, as encountered by a counterclockwise radial sweep around $p$. Consider the closed polygonal chain $P=\left(p_{1}, \ldots, p_{n}\right)$ and observe that $P$ describes the boundary of a simple polygon (Fig. 8). With $\angle p q r$ denote the counterclockwise angle between the edges $p q$ and $q r$ around $q$. A point $p_{i}, 2 \leq i<n$, is reflex if the interior angle of $P$ at $p_{i}$ is reflex, that is, $\angle p_{i-1} p_{i} p_{i+1}>\pi$; otherwise, $p_{i}$ is convex. Thus, $p_{1}, p_{2}$, and $p_{n}$ are convex.

We construct a triangulation $T$ on $S$ as follows. As a start, we take the edges of $\mathrm{CH}(S)$ and all edges of $P$, and denote the resulting graph by $T_{0}$. If $P$ is convex then $T_{0}$ forms a convex polygon. Otherwise $\mathrm{CH}(S)$ is partitioned into two or more faces by the edges of $P$. Thinking of $p$ as a light source and of $P$ as opaque, we call the face of $T_{0}$ that contains $p$ the light face and the other faces of $T_{0}$ dark faces. Dark faces are shown gray in figures. 
In a next step, we insert further edges to ensure that all faces are convex. The light face is made convex by adding all edges $p p_{i}$ where $p_{i}$ is reflex. Hence the light face of $T_{0}$ might be split into a number of faces, all of which we refer to as light faces in the following. We partition the dark faces into convex faces as follows. First, we add all edges to connect the subsequence of $P$ that consists of all convex points by a polygonal path. Note that some of those edges may be edges of $P$ or $\mathrm{CH}(S)$ and, hence, already be present. Next, we triangulate those dark faces that are not convex. For now, let us say that these faces are triangulated arbitrarily. Later, we add a little twist.

Our construction is based on choosing particular triangulations for those faces that share at least two consecutive edges with $P$. Let us refer to these faces as interesting, while the remaining ones are called uninteresting. The interesting faces can be ordered linearly along $P$, such that any two successive faces share exactly one edge. We denote this order by $f_{1}, \ldots, f_{m}$. Note that $f_{i}$ is light for $i$ odd and dark for $i$ even, and that both $f_{1}$ and $f_{m}$ are light. Also observe that $p$ is a vertex of every light face; therefore, any interesting light face other than $f_{1}$ and $f_{m}$ has at least four vertices and all uninteresting light faces are triangles. On the dark side, however, there may be both interesting triangles and uninteresting faces with more than three vertices. Similar to above, we triangulate all uninteresting dark faces, for now, arbitrarily (a little twist will come later). We denote the resulting graph by $T_{1}$.

As a final step, we triangulate the interesting faces $f_{1}, \ldots, f_{m}$ of $T_{1}$ in this order to obtain a triangulation on $S$ with the desired happiness ratio. We always treat a light face $f_{i}$ and the following dark face $f_{i+1}$ together. The vertices that do not occur in any of the remaining faces are removed, and the goal is to choose a local triangulation for $f_{i}$ and $f_{i+1}$ that makes a large fraction of those vertices happy. The progress is measured by the happiness ratio $h / t$, if $h$ vertices among $t$ removed vertices are happy. Note that these ratios are similar to fractions. But in order to determine the collective happiness ratio of two successive steps, the corresponding ratios have to be added component-wise. In that view, for instance, $2 / 2$ is different from $3 / 3$.

We say that some set of points can be made happy "using a face $f$ ", if $f$ can be triangulated-for instance using Corollary 1 or Observation 1 -such that all these points are happy. Two vertices are aligned, if either both are currently happy or both are currently unhappy. Two vertices that are not aligned are contrary. Denote the boundary of a face $f$ by $\partial f$, and let $\partial f_{i}=\left(p, p_{j}, \ldots, p_{k}\right)$, for some $k \geq j+2$, and $\partial f_{i+1}=\left(p_{k-1}, \ldots, p_{r}\right)$, for some $r \geq k+1$.

After treating $f_{i}$ and $f_{i+1}$, we have removed all vertices up to, but not including, the last two vertices $p_{r-1}$ and $p_{r}$ of $f_{i+1}$, which coincide with the first two vertices of the next face $f_{i+2}$. Sometimes, the treatment of $f_{i}$ and $f_{i+1}$ leaves the freedom to vary the parity of the vertex $p_{r-1}$ while maintaining the desired happiness ratio as well as the parity of $p_{r}$. This means that the future treatment of $f_{i+2}$ and $f_{i+3}$ does not need to take care of the parity of $p_{r-1}$. By adjusting the triangulation of $f_{i}$ and $f_{i+1}$ we can always guarantee that $p_{r-1}$ is happy.
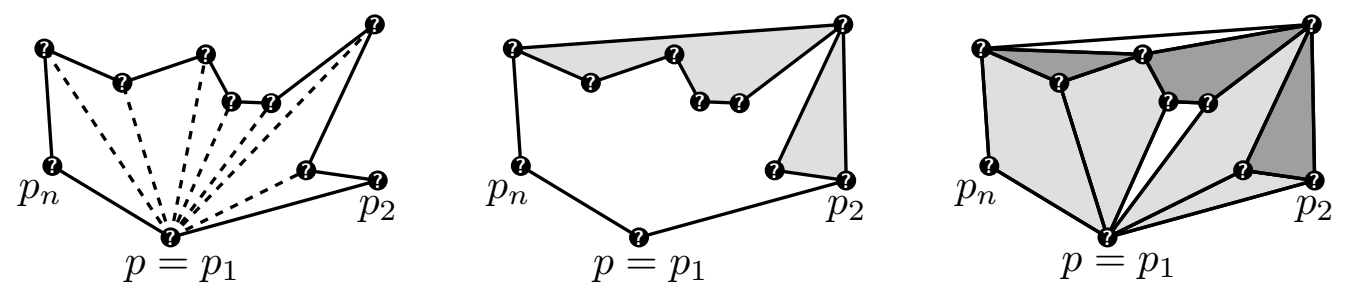

Fig. 8 The simple polygon bounded by $P$, the initial graph $T_{0}$ (with dark faces shown gray), and the graph $T_{1}$ in which all faces are convex (interesting light and dark faces shown light gray and dark gray, respectively). 
Therefore, we distinguish two different settings regarding the treatment of a face pair: no choice (the default setting with no additional help from outside) and $1^{\text {st }}$ choice (we can flip the parity of the first vertex $p_{j}$ of the face and, thus, always make it happy).

No choice. We distinguish cases according to the number of vertices in $f_{i}$.

(1.1) $k \geq j+3$, that is, $f_{i}$ has at least five vertices. Then $p_{j}, \ldots, p_{k-2}$ can be made happy using $f_{i}$, and $p_{k-1}, \ldots, p_{r-3}$ can be made happy using $f_{i+1}$. Out of the $r-j-1$ points removed, at least $(k-2-j+1)+(r-3-(k-1)+1)=$ $r-j-2$ are happy. As $r-j \geq 4$, this yields a happiness ratio of at least $2 / 3$. The figure to the right shows the case

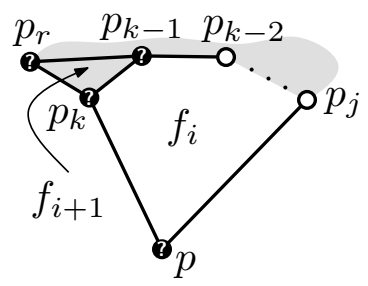
$r=k+1$ as an example.

(1.2) $k=j+2$, that is, $f_{i}$ is a convex quadrilateral. We distinguish subcases according to the number of vertices in $f_{i+1}$.

(1.2.1) $r \geq j+4$, that is, $f_{i+1}$ has at least four vertices. Using $f_{i+1}$, all of $p_{j+3}, \ldots, p_{r-2}$ can be made happy. Then at least two out of $p_{j}, \ldots, p_{j+2}$ can be made happy using $f_{i}$. Overall, at least $r-2-(j+$ $3)+1+2=r-j-2$ out of $r-j-1$ removed points are happy. As $r-j \geq 4$, the happiness ratio is at least $2 / 3$.

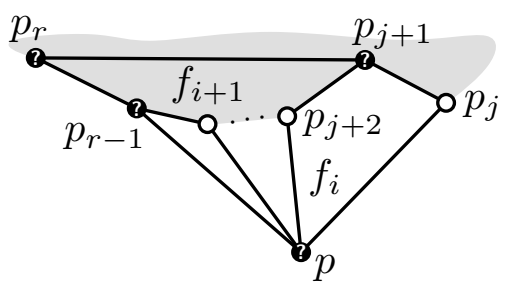

(1.2.2) $r=j+3$, that is, $f_{i+1}$ is a triangle. If both $p_{j}$ and $p_{j+1}$ can be made happy using $f_{i}$, the happiness ratio is $2 / 2$. Otherwise, regardless of how $f_{i}$ is triangulated ex-
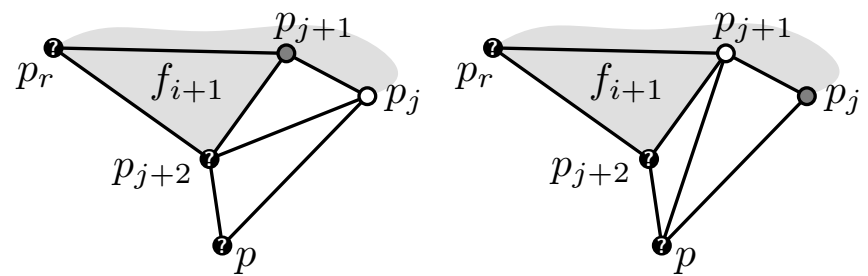
actly one of $p_{j}$ and $p_{j+1}$ is happy, see the figure to the right. This yields a ratio of $1 / 2$ and $1^{\text {st }}$ choice for $f_{i+2}$.

First choice. Denote by $f^{\prime}$ the other (than $f_{i}$ ) face incident to the edge $p_{j} p_{j+1}$ in the current graph. As all of $f_{1}, \ldots, f_{i-1}$ are triangulated already, $f^{\prime}$ is a triangle whose third vertex (other than $p_{j}$ and $p_{j+1}$ ) we denote by $p^{\prime}$. Recall that in the $1^{\text {st }}$ choice setting we assume that, regardless of how $f_{i}$ is triangulated, $p_{j}$ can be made happy. More precisely, we assume the following in a $1^{\text {st }}$ choice scenario with a face pair $f_{i}, f_{i+1}$ to be triangulated: By adjusting the triangulations of $f_{1}, \ldots, f_{i-1}$, we can synchronously flip the parity of both $p_{j}$ and $p^{\prime}$, such that

(C1) All faces $f_{i}, f_{i+1}, \ldots, f_{m}$ as well as $f^{\prime}$ remain unchanged,

(C2) the degree of all of $p_{j+1}, \ldots, p_{n}$ remains unchanged, and

(C3) the number of happy vertices among $p_{2}, \ldots, p_{j-1}$ does not decrease.

Observe that these conditions hold after Case 1.2.2. Using this $1^{\text {st }}$ choice flip, we may suppose that $p^{\prime}$ is happy. Then by (C3) the number of happy vertices among $\left\{p_{2}, \ldots, p_{j-1}\right\} \backslash$ $\left\{p^{\prime}\right\}$ does not decrease, in case we do the $1^{\text {st }}$ choice flip (again) when processing $f_{i}, f_{i+1}$. We distinguish cases according to the number of vertices in $f_{i}$. 
(2.1) $k \geq j+3$, that is, $f_{i}$ has at least five vertices. Then $p_{j+1}, \ldots, p_{k-1}$ can be made happy using $f_{i}$. If $f_{i+1}$ is a triangle (as shown in the figure to the right), this yields a ratio of at least $3 / 3$. Otherwise $(r \geq k+2)$, apart from keeping $p_{k-1}$ happy, $f_{i+1}$ can be used to make all of $p_{k}, \ldots, p_{r-3}$ happy. At least $r-j-2$ out of $r-j-1$ vertices removed are happy,

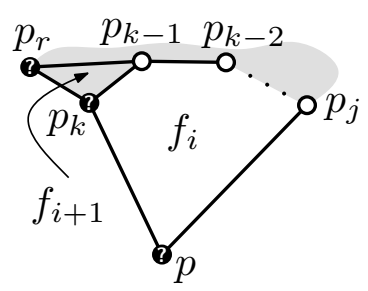
for a happiness ratio of at least $3 / 4$.

(2.2) $k=j+2$, that is, $f_{i}$ is a convex quadrilateral. We distinguish subcases according to the size of $f_{i+1}$.

(2.2.1) $r \geq j+5$, that is, $f_{i+1}$ has at least five vertices. Triangulate $f_{i}$ arbitrarily and use $f_{i+1}$ to make all of $p_{j+1}, \ldots, p_{r-3}$ happy. At least $r-j-2$ out of $r-j-1$ vertices removed are happy, for a happiness ratio of at least $3 / 4$.

(2.2.2) $r=j+3$, that is, $f_{i+1}$ is a triangle. Use $f_{i}$ to make $p_{j+1}$ happy for a perfect ratio of $2 / 2$.

(2.2.3) $r=j+4$, that is, $f_{i+1}$ is a convex quadrilateral. If $p_{j+1}$ and $p_{j+2}$ are aligned, then triangulating $f_{i}$ arbitrarily makes them contrary. Using $f_{i+1}$ both can be made happy, for a perfect $3 / 3$ ratio overall. Thus, suppose that $p_{j+1}$ and $p_{j+2}$ are contrary. We make a further case distinction according to the position of $p_{j}$ with respect to $f_{i+1}$.

(2.2.3.1) $\angle p_{j+3} p_{j+2} p_{j} \leq \pi$, that is, $p, p_{j}, p_{j+2}, p_{j+3}$ form a convex quadrilateral. Add edge $p_{j} p_{j+2}$ and exchange edge $p p_{j+2}$ with edge $p_{j} p_{j+3}$. In this way, $p_{j+1}$ and $p_{j+2}$ remain contrary. Hence, both $p_{j+1}$ and $p_{j+2}$ can be made happy using $f_{i+1}$, for a perfect ratio of $3 / 3$ overall.
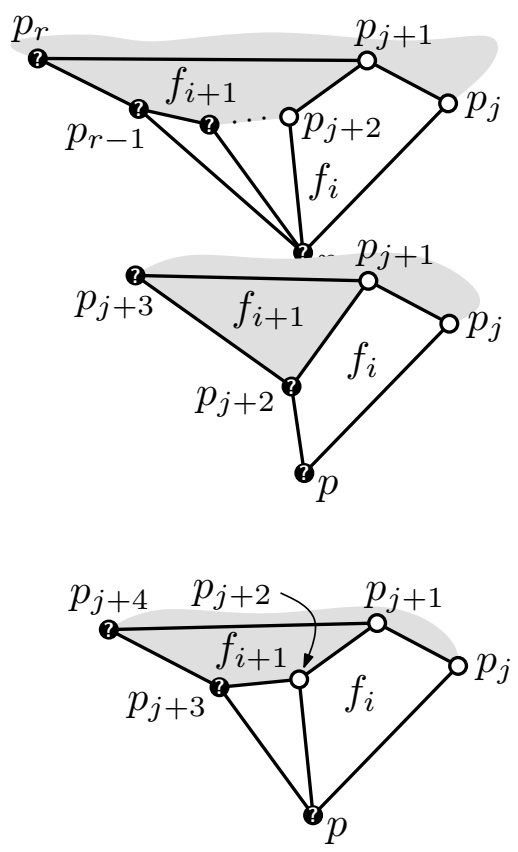

(2.2.3.2) $\angle p_{j} p_{j+1} p_{j+3} \leq \pi$, that is, the points $p_{j}, p_{j+4}, p_{j+3}, p_{j+1}$ form a convex quadrilateral. To conquer this case we need $p^{\prime} p_{j+4}$ to be an edge of $T_{1}$. In order to ensure this, we apply the before mentioned little twist: before triangulating the nonconvex dark faces, we scan through the sequence of dark faces for configurations of

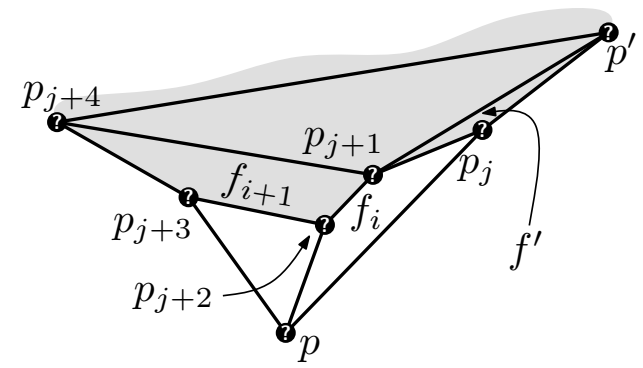
points like in this case. Call a dark quadrilateral $f_{i}$ with $\partial f_{i}=\left(p_{j+1}, \ldots, p_{j+4}\right)$ delicate if $\angle p_{j} p_{j+1} p_{j+3} \leq \pi$. For every delicate dark quadrilateral $f_{i}$ in $f_{4}, f_{6}, \ldots, f_{m-1}$ such that $f_{i-2}$ is not delicate, add the edge $p_{j+4} p_{h}$, where $p_{h}$ is the first vertex of $f_{i-2}$. Observe that this is possible as $p_{h}, \ldots, p_{j+1}, p_{j+3}, p_{j+4}$ form a convex polygon $f^{*}: p_{h}, \ldots, p_{j+1}$ and $p_{j+1}, p_{j+3}, p_{j+4}$ form convex chains being vertices of $f_{i-2}$ and $f_{i}$, respectively, and $p_{j+1}$ is a convex vertex of $f^{*}$ because $\angle p_{j} p_{j+1} p_{j+3} \leq \pi$. Then we triangulate the remaining non-convex and the uninteresting dark faces arbitrarily to get $T_{1}$. 
To handle this case we join $f_{i+1}$ with $f^{\prime}$ by removing the edges $p_{j+1} p_{j+4}$ and $p^{\prime} p_{j+1}$ and adding the edge $p_{j+3} p_{j+1}$, which yields a convex pentagon $f^{*}=$ $p_{j+4}, p_{j+3}, p_{j+1}, p_{j}, p^{\prime}$. Observe that $p_{j+1}$ and $p_{j+2}$ are aligned now. Thus, making $p_{j+2}$ happy using $f_{i}$ leaves $p_{j+1}$ unhappy. If $p^{\prime}$ and $p_{j}$ are aligned, then triangulate $f^{*}$

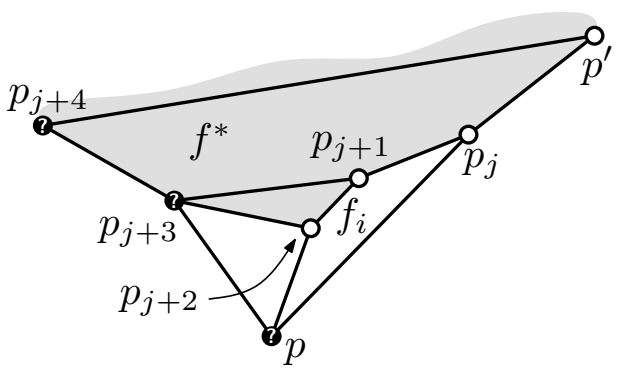
using a star from $p^{\prime}$, making $p_{j+1}$ happy. As $p^{\prime}$ and $p_{j}$ remain aligned, both can be made happy-possibly using the $1^{\text {st }}$ choice flip—for a perfect $3 / 3$ ratio. If, on the other hand, $p^{\prime}$ and $p_{j}$ are contrary, then triangulate $f^{*}$ using a star from $p_{j+4}$, making $p_{j+1}$ happy. Now $p^{\prime}$ and $p_{j}$ are aligned and both can made happy-possibly using the $1^{\text {st }}$ choice flip-for a perfect $3 / 3$ ratio.

(2.2.3.3) Neither of the previous two cases occurs and, thus, $p_{j}, p_{j+1}, p_{j+3}, p_{j+2}$ form a convex quadrilateral $f^{*}$. Remove $p_{j+1} p_{j+2}$ and add $p_{j+1} p_{j+3}$ and $p_{j} p_{j+2}$. Note that $p_{j}$ is happy because of $1^{\text {st }}$ choice for $f_{i}$, and $p_{j+1}$ and $p_{j+2}$ are still contrary. Therefore, independent of the triangulation of $f^{*}$, at least two vertices out

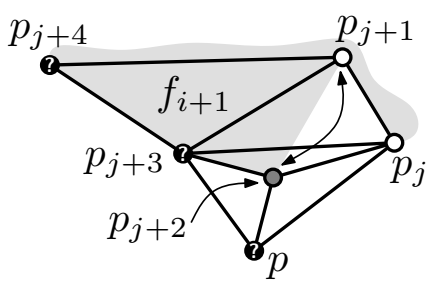
of $p_{j}, p_{j+1}, p_{j+2}$ are happy. Moreover, using $f^{*}$ we can synchronously flip the parity of both $p_{j+1}$ and $p_{j+3}$ such that $(\mathrm{C} 1)-(\mathrm{C} 3)$ hold. This gives us a ratio of $2 / 3$ and $1^{\text {st }}$ choice for $f_{i+2}$.

Putting things together. Recall that the first face $f_{1}$ and the last face $f_{m}$ are the only light faces that may be triangles. In case that $f_{1}$ is a triangle, we just accept that $p_{2}$ may stay unhappy, and using $f_{2}$ the remaining vertices removed, if any, can be made happy. Similarly, from the last face $f_{m}$ up to three vertices may remain unhappy. To the remaining faces $f_{3}, \ldots, f_{m-1}$ we apply the algorithm described above.

In order to analyze the overall happiness ratio, denote by $h_{0}(n)$ the minimum number of happy vertices obtained by applying the algorithm described above to a sequence $P=$ $\left(p_{1}, \ldots, p_{n}\right)$ of $n \geq 3$ points in a no choice scenario. Similarly, denote by $h_{1}(n)$ the minimum number of happy vertices obtained by applying the algorithm described above to a sequence $P=\left(p_{1}, \ldots, p_{n}\right)$ of $n \geq 3$ points in a $1^{\text {st }}$ choice scenario. From the case analysis given above we deduce the following recursive bounds.

a) $h_{0}(n)=0$ and $h_{1}(n)=1$, for $n \leq 4$.

b) $h_{0}(n) \geq \min \left\{2+h_{0}(n-3), 1+h_{1}(n-2)\right\}$.

c) $h_{1}(n) \geq \min \left\{3+h_{0}(n-4), 2+h_{0}(n-2), 2+h_{1}(n-3)\right\}$.

By induction on $n$ we can show that $h_{0}(n) \geq\lceil(2 n-8) / 3\rceil$ and $h_{1}(n) \geq\lceil(2 n-7) / 3\rceil$. Taking the at most four unhappy vertices from $f_{1}$ and $f_{m}$ into account yields the claimed overall happiness ratio.

\section{Conclusion}

In this paper we considered the construction of crossing-free geometric graphs on point sets with constraints on the parity of the vertex degrees. For all but at most three vertices the constraints can be fulfilled when constructing outerplanar graphs and pointed pseudotriangulations. For triangulations, we showed that there can be a linear number of such 
vertices and gave a construction that allows making $\left\lfloor\frac{2 n}{3}\right\rfloor-6$ vertices happy. For polygons with polygonal holes, we proved the according decision problem to be NP-complete.

For the case where all vertices are labeled odd, Proposition 2 showed that one can achieve a fraction $\frac{10}{13}$ of happy vertices. There might be ways to further improve this constant factor. We even conjecture that this factor is 1 , that is, every planar point set has a triangulation with at most $K$ even vertices, for some absolute constant $K$.

Acknowledgements We thank Wolfgang Aigner, Franz Aurenhammer, Markus Demuth, Elena Mumford, Davíd Orden, and Pedro Ramos for fruitful discussions.

\section{References}

1. O. Aichholzer, T. Hackl, M. Hoffmann, A. Pilz, G. Rote, B. Speckmann, and B. Vogtenhuber. Plane graphs with parity constraints. In F. Dehne, I. Munro, J.-R. Sack, and R. Tamassia, editors, Algorithms and Data Structures Symposium - WADS 2009, volume 5664 of Lecture Notes in Computer Science, pages 13-24. Springer, 2009.

2. O. Aichholzer, T. Hackl, C. Huemer, F. Hurtado, and B. Vogtenhuber. Large bichromatic point sets admit empty monochromatic 4-gons. SIAM Journal on Discrete Mathematics, 23(4):2147-2155, 2010.

3. O. Aichholzer and H. Krasser. The point set order type data base: A collection of applications and results. In Proc. 13th Canadian Conference on Computational Geometry, pages 17-20, Waterloo, Ontario, Canada, 2001.

4. V. Alvarez. Even triangulations of planar sets of points with Steiner points. In Proc. 26th European Workshop on Computational Geometry - EuroCG 2010, pages 37-40, Dortmund, Germany, 2010.

5. P. Bose. On embedding an outer-planar graph in a point set. Computational Geometry: Theory and Applications, 23(3):303-312, 2002.

6. P. Bose, M. McAllister, and J. Snoeyink. Optimal algorithms to embed trees in a point set. Journal of Graph Algorithms and Applications, 1(2):1-15, 1997.

7. C. Colbourn and K. Booth. Linear time automorphism algorithms for trees, interval graphs, and planar graphs. SIAM Journal on Computing, 10(1):203-225, 1981.

8. P. Erdős and T. Gallai. Graphs with prescribed degree of vertices. Mat. Lapok, 11:264-274, 1960.

9. I. Fernández Delago, C. I. Grima Ruiz, A. Márquez Pérez, A. Nakamoto, R. Robles Arias, and J. Valenziela Munõz. Even and quasi-even triangulations of point sets in the plane. In Proc. 26th European Workshop on Computational Geometry - EuroCG 2010, pages 161-164, Dortmund, Germany, 2010.

10. H. Fleischner. Gedanken zur Vier-Farben-Vermutung. Monatshefte für Mathematik, 79(3):201-211, 1975.

11. P. D. Gilbert. New results on planar triangulations. Master's thesis, University of Illinois at UrbanaChampaign, 1979.

12. K. Jansen. One strike against the min-max degree triangulation problem. Computational Geometry: Theory and Applications, 3(2):107-120, 1993.

13. L. Kettner, D. Kirkpatrick, A. Mantler, J. Snoeyink, B. Speckmann, and F. Takeuchi. Tight degree bounds for pseudo-triangulations of points. Computational Geometry: Theory and Applications, 25(1\&2):1-12, 2003.

14. G. T. Klincsek. Minimal triangulations of polygonal domains. Annals of Discrete Mathematics, 9:121123, 1980.

15. A. Kooshesh and B. Moret. Folding a triangulated simple polygon: Structural and algorithmic results. In F. Dehne, F. Fiala, and W. W. Koczkodaj, editors, Proc. of the International Conference on Computing and Information, ICCI '91, volume 497 of Lecture Notes in Computer Science, pages 102-110. Springer, 1991.

16. D. Lichtenstein. Planar formulae and their uses. SIAM Journal on Computing, 11(2):329-343, 1982.

17. J. O'Rourke. Computational Geometry in C. Cambridge University Press, New York, NY, USA, $2^{\text {nd }}$ edition, 1998.

18. A. Pilz. Parity properties of geometric graphs. Master's thesis, Graz University of Technology, Austria, 2009.

19. A. Tamura and Y. Tamura. Degree constrained tree embedding into points in the plane. Information Processing Letters, 44:211-214, 1992. 